\title{
Erratum to: Editorial: Multimedia in Technology Enhanced Learning
}

\author{
Zhigao Zheng ${ }^{1} \cdot$ Jinming Wen ${ }^{2} \cdot$ Shuai Liu ${ }^{3}$
}

Published online: 8 February 2017

(C) Springer Science+Business Media New York 2017

\section{Erratum to: Mobile Netw Appl \\ DOI 10.1007/s11036-016-0798-1}

The original version of this article was revised. Below paragraphs were removed:

In the sixth paper "A Novel Hybrid Approach Based On Chaotic Cloud Gravitational Search Algorithm to Complicated Image Template Matching", the author put forward a novel chaotic cloud search mechanism based Gravitational Search Algorithm (CCGSA) for the template matching of digital images. Gravitational Search Algorithm (GSA) is a new type smart algorithm derived from universal gravitation. To further strengthen the optimizing capacity of GSA, a cross search strategy based on chaotic global search (CGS) and cloud local search (CLS) is incorporated into GSA. CGS improves the ability of global search of GSA and effectively prevents the premature phenomenon by using the ergodicity of chaos. CLS conducts a fine search around the current best location according to the randomness and stable tendency of the normal cloud model, which greatly improves the convergence accuracy and accelerates the convergence velocity. The numerical imitation results reveal that our proposed algorithm has more significant advantages in solving template matching problems than the other intelligent optimization algorithms.

The last article "A Family of High-Order Newton's Iterative Methods in Image Enhancement" proposed a family of thirdorder iterative methods to improve the current image enhancement methods. This method uses only two function calculations and an equation derivation in each iterative process. When the parameter $\beta$ of this method is set as 0.5 , the fourth-order convergence of the iterative method can be achieved, indicating higher efficiency than some classical approach. In addition, the family of third-order iterative methods are adopted to construct a new multi-step iterative method of high-order. By comparison and analysis of several examples, the new two-step iterative methods can quickly approximate to the root of the equations after several iterations and indicate higher computational efficiency.

The online version of the original article can be found at http://dx.doi. org/10.1007/s11036-016-0798-1

\author{
Zhigao Zheng \\ zhengzhigao@pku.edu.cn \\ Jinming Wen \\ jinming.wen@ens-lyon.fr \\ Shuai Liu \\ cs_liushuai@imu.edu.cn \\ 1 Central China Normal University, Wuhan, China \\ 2 Centre national de la recherché scientifique, Paris, France \\ 3 Inner Mongolia University, Hohhot, China
}

